\title{
THE COHOMOLOGY OF THE MODULI SPACE OF CURVES
}

The purpose of this unit is to give a brief survey about the cohomology and the tautological rings of the moduli space of curves. Unfortunately, for the most part the cohomology of $M_{g}$ remains mysterious. Some of the outstanding problems include:

Problem 0.1. What is the cohomological dimension of $M_{g}$ ?

Problem 0.2. Which classes generate the cohomology of $M_{g}$ ?

Problem 0.3. What are the largest dimensional subvarieties of $M_{g}$ ? What are the largest dimensional subvarieties of $M_{g}$ that pass through a general point?

These are a few problems from an endless list of unknowns about the cohomology of $M_{g}$. Despite our ignorance there has been a tremendous effort to study the cohomology of $M_{g}$ in the last three decades. Especially Harer and his students using cohomological methods applied to the mapping class group have obtained major results about the cohomology of $M_{g}$. In this unit we will survey some of these results.

\section{TeICHMÜLLER THEORY}

There are many good references for Teichmüller theory. Two books that are friendly to algebraic geometers are [IT and [Ab. A more analytic introduction can be found in [Le]. Curt McMullen regularly teaches courses on the subject and he has really nice course notes on the subject.

From now on we will be working over the complex numbers $\mathbb{C}$. Accordingly we will use the Let $S$ be a closed, oriented surface of genus $g$.

Definition 1.1. A marked Riemann surface is a pair $(R,[f])$ consisting of a Riemann surface and the homotopy class of an oriantation preserving homeomorphism $f: R \rightarrow S$. Two marked Riemann surfaces $\left(R_{1},\left[f_{1}\right]\right),\left(R_{2}, f_{2}\right)$ are equivalent if there exists a holomorphic map $h: R_{1} \rightarrow R_{2}$ such that $\left[f_{2} \circ h\right]=\left[f_{1}\right]$.

Definition 1.2. The set of equivalence classes of marked Riemann surfaces is called the Teichmüller space $T_{g}$ of genus $g$. 
We will shortly describe the Fenchel-Nielsen coordinates on Teichmüller space. Under the topology induced by these coordinates $T_{g}$ becomes homeomorphic to $\mathbb{R}^{6 g-g}$; hence it is a contractible space. On the other hand, $T_{g}$ is very closely related to $M_{g}$. Certainly if $\left(R_{1}, f_{1}\right)$ is equivalent to $\left(R_{2}, f_{2}\right)$, then $R_{1}$ and $R_{2}$ have to be isomorphic as Riemann surfaces. This suggests that the moduli space $M_{g}$ should be a quotient of $T_{g}$.

Definition 1.3. Define the mapping class group $\Gamma_{g}$ of genus $g$ to be the isotopy classes of orientation preserving homeomorphisms of $F$.

$$
\begin{aligned}
& \Gamma_{g} \text { acts on } T_{g} \text { by } \\
& \qquad[w]_{*}(R,[f])=(R,[w \circ f]) \quad \text { for }[w] \in \Gamma_{g} .
\end{aligned}
$$

This action of $\Gamma_{g}$ on $T_{g}$ is properly discontinuous: for any two compact subsets $K_{1}$ and $K_{2}$ of $T_{g}$ there exists only finitely many $[w] \in \Gamma_{g}$ for which $K_{1} \cap[w]_{*} K_{2} \neq \emptyset$. If $\left(R_{1},\left[f_{1}\right]\right)$ and $\left(R_{2},\left[f_{2}\right]\right)$ become identified under the action of $\Gamma_{g}$, then $R_{1}$ and $R_{2}$ are isomorphic as Riemann surfaces. Conversely, if there is an isomorphism $h: R_{1} \rightarrow R_{2}$ between $R_{1}$ and $R_{2}$, we get an element of $\Gamma_{g}$ by taking $\left[f_{2} \circ h \circ f_{1}^{-1}\right.$. In fact $T_{g}$ may be embedded in $\mathbb{C}^{3 g-3}$ as a bounded, contractible domain by the Bers embedding. Once we know this fact, we obtain a new construction of $M_{g}$ as a complex orbifold.

In the sequel we will frequently invoke the Uniformization Theorem from elementary complex analysis, so we recall its statement.

Theorem 1.4 (Uniformization). Any simply connected Riemann surface is biholomorphic to the Riemann sphere $\hat{\mathbb{C}}$, the complex plane $\mathbb{C}$ or the upper half-plane $\mathbb{H}$. Furthermore, among $\hat{\mathbb{C}}, \mathbb{C}$ and $\mathbb{H}$ no two are mutually biholomorphic.

Example 1.5. The uniformization theorem together with the following theorem from elementary topology

Theorem 1.6. Any orientation preserving homeomorphism of the twodimensional sphere to itself is isotopic to the identity.

we conclude that $T_{0}$ consists of a single point.

Example 1.7. The universal cover of a Riemann surface naturally has the structure of a Riemann surface. The uniformization theorem implies that the universal cover of a genus one Riemann surface is the complex plane. Every genus one Riemann surface is the quotient of the complex plane by a rank 2 lattice. We can normalize the lattice so 
that it is generated by $(1, \tau)$ where $\tau \in \mathbb{H}$. It is well known that two complex tori represented by $\tau$ and $\tau^{\prime}$ are biholomorphic if and only if there exists $M \in \mathbb{P} S L_{2}(\mathbb{Z})$ such that $\tau=M \tau^{\prime}$. If in addition, we want the map to be isotopic to the identity, we can assume that in the lift of the map 1 maps to 1 . It follows that then two tori represent the same point in $T_{1}$ if and only if $\tau=\tau^{\prime}$. Thus we identify $T_{1}$ with $\mathbb{H}$.

Now that we have described the spaces $T_{0}$ and $T_{1}$, we will assume that $g \geq 2$. Every subgroup of $\operatorname{Aut}(\mathbb{C})$ that acts properly discontinuously and fixed point free on $\mathbb{C}$ is abelian. It follows that any Riemann surface of genus at least two is uniformized by the upper half plane. The upper half plane with the Poincaré metric is also a model for hyperbolic two space. Since the Poincaré metric on $\mathbb{H}$ is invariant under $\mathbb{P} S L_{2}(\mathbb{R})$, hence Riemann surfaces of genus $g>1$ inherit a hyperbolic metric. Furthermore, two Riemann surfaces of genus $g>1$ are conformally equivalent if and only if they are isometric with respect to the hyperbolic metric they inherit from $\mathbb{H}$ since $\mathbb{P} S L_{2}(\mathbb{R})$ is both the group of conformal self maps of $\mathbb{H}$ and the isometries of the Poincaré metric on $\mathbb{H}$. We thus obtain a different description of the Teichmüller space for hyperbolic surfaces. Consider marked hyperbolic surfaces $(X,[f])$, where $X$ is a smooth, oriented surface equipped with a complete Riemannian metric of constant curvature -1 and $[f]$ is the homotopy class of an orientation preserving homeomorphism $f: X \rightarrow F$ to a fixed compact, oriented genus $g$ surface $F$. Two marked hyperbolic surfaces $\left(X_{1},\left[f_{1}\right]\right)$ and $\left(X_{2},\left[f_{2}\right]\right)$ are equivalent if there exists an isometry $h: X_{1} \rightarrow X_{2}$ such that $\left[f_{2} \circ h\right]=\left[f_{1}\right]$. The resulting space is again the Teichmüller space $T_{g}$ of genus $g$.

We next introduce the Fenchel-Nielsen coordinates on Teichmüller space. Given a hyperbolic surface of genus $g>1$, we can cut the surface along $3 g-3$ simple, closed curves to obtain $2 g-2$ pieces called pants that are homeomorphic to a disc with two smaller inner discs removed. In a hyperbolic surface each free-homotopy (equivalently isotopy) class contains a unique geodesic loop. Moreover, if $\alpha_{1}, \cdots, \alpha_{n}$ are pair wise non-isotopic, non-intersecting simple loops, then we can find non-intersecting simple closed geodesics $\gamma_{1}, \cdots, \gamma_{n}$ with $\gamma_{i}$ isotopic to $\alpha_{i}$. The $\gamma_{i}$ are uniquely determined. Therefore, we can assume that we are cutting the surface along geodesics. Note that the conformal structure of the pants is determined by the lengths of the ordered (geodesic) boundary components of the pants. Consider a Fuchsian model for the surface. Then the stabilizer of a connected component of the inverse image of the pants is a free subgroup $G_{P}$ of $A u t(\mathbb{H})$ generated by two 
hyperbolic elements. We can realize the pants as the quotient of the upper half plane by $G_{P}$. We can conjugate $G_{P}$ so that its generators are of the form

$g_{1}=(\lambda)^{2} z, 0<\lambda<1, \quad g_{2}=\frac{a z+b}{c z+d}, a d-b c=1, a+d=b+c, c>0$

and we can assume that $g_{1}, g_{2}$ and $\left(g_{2} \circ g_{1}\right)^{-1}$ cover the boundary geodesics $\gamma_{1}, \gamma_{2}, \gamma_{3}$, respectively. There is a useful relation between the length of $\gamma_{i}$ and the square of the trace of $g_{i}$.

Lemma 1.8. Let $R$ be a Riemann surface uniformized by $\mathbb{H}$. Let $g$

$$
g(z)=\frac{a z+b}{c z+d}, a d-b c=1
$$

be a hyperbolic element in the Fuchsian group of $R$ and let $\gamma$ be the closed geodesic on $R$ corresponding to $g$. Then

$$
\operatorname{tr}^{2}(g)=4 \cosh ^{2}\left(\frac{l(\gamma)}{2}\right)
$$

Proof. Since both sides of the equation are invariant under conjugation, we can assume that $g=\lambda z$ with $\lambda>1$. The length of the geodesic covered by $g$ is given by

$$
\int_{1}^{\lambda} \frac{d y}{y}=\log \lambda
$$

The lemma follows from the equality

$$
(\sqrt{\lambda}+1 / \sqrt{\lambda})^{2}=4 \cosh ^{2}\left(\frac{\log \lambda}{2}\right) .
$$

By equation (1) the lengths of the boundary components of the pants determine the generators of $G_{P}$ and consequently the conformal structure of the pants.

Next, observe that given any two boundary components of the pants, there is a unique geodesic that intersects both perpendicularly. Join the two boundary components by an arc. Double the pants to obtain a hyperbolic genus 2 surface. The arcs form a simple closed loop, hence there is a unique simple closed geodesic in the isotopy class of the loop. By symmetry this geodesic must intersect the boundary of the pants perpendicularly. The intersection point of this geodesic with a boundary component serves as a reference point to measure any twisting in the boundary when gluing two pants. The ordered pair of lengths of the $3 g-3$ geodesics and the $3 g-3$ twist parameters give a map from $T_{g}$ into $\left(\mathbb{R}^{+}\right)^{3 g-3} \times(\mathbb{R})^{3 g-3}$. This is in fact a bijection. We thus obtain a topology on $T_{g}$ under which $T_{g}$ is homeomorphic to 
a contractible open subset of $\mathbb{R}^{6 g-6}$. Let us briefly recall some of the ingredients of the proof.

Lemma 1.9. Given an arbitrary ordered triple of positive real numbers $\left(l_{1}, l_{2}, l_{3}\right)$ there exists a right hexagon in the hyperbolic plane such that the length of every other side is equal to $l_{i}$.

Proof. Consider the unit circle model of the hyperbolic plane. Fix a portion $C_{1}$ of the imaginary axis. Find another geodesic $C_{2}$ such that the distance from $C_{1}$ to this geodesic is $l_{1}$. There is a one parameter family of geodesics a distance $l_{2}$ away from $C_{1}$, hence one that is a distance $l_{3}$ away from $C_{2}$. This gives the desired right hexagon. Observe that by letting one or two of the lengths go to zero, we obtain an ideal pentagon and an ideal quadrilateral, respectively. This will be important later when we generalize the discussion to Riemann surfaces with marked points.

The right hexagons are closely related to the pants decomposition of surfaces. If we take a pair of pants with geodesic boundaries and cut them along the perpendicular geodesics that join the boundaries pair wise, we obtain a pair of equivalent right hexagons. (Observe that two perpendicular geodesics that join different boundary components are disjoint.) Conversely, to obtain a pair of pants identify every other side of two equivalent right hexagons. When identifying the hexagons, give them opposite orientations and glue them along their boundaries respecting the orientation. The correspondence between pants and hexagons together with Lemma 1.9 allows us to conclude that there is a pair of pants with any ordered triple of positive numbers as the lengths of its boundary components. Moreover, the conformal structure of the pants is uniquely determined by these lengths. We obtain a surjective map from Teichmüller space to $\left(\mathbb{R}^{+}\right)^{3(g-1)}$. Fix $3(g-1)$ simple closed curves $\alpha_{i}$ (see Figure 1) that cut the surface into pants. As we vary the hyperbolic metric on the surface the lengths of the geodesics in the isotopy classes of these simple closed curves assume all values in $\left(\mathbb{R}^{+}\right)^{3(g-1)}$. Observe that we can glue two pants (metrically) along two geodesic boundary components if and only if the boundary components have the same length. We identify two points on the boundary and then glue according to arc length. But we can also twist the boundary components by an angle before we glue. There remains to show that any twisting in any of the curves leads to different points of Teichmüller space. For the proof one fixes a non-trivial loop $\beta_{i}$ that intersects $\alpha_{i}$ at two points. Then there is a function from $\mathbb{R}^{3(g-1)}$ to $\mathbb{R}$ given by the length of the geodesic loop in the homotopy class of $\beta_{i}$ as one varies the 
metric by twisting the $3(g-1)$ curves. One proves that the functions so obtained are strictly convex and have minima. This implies then that there is a bijection between Teichmüller space and $\left(\mathbb{R}^{+} \times \mathbb{R}\right)^{3(g-1)}$.

Figure 1. Cutting a genus 2 surface into pairs of pants.

Remark 1.10. It is possible to give a third definition of Teichmüller space in terms of quasiconformal mappings. Using this point of view one can introduce a complex structure on Teichmüller space so that $T_{g}$ becomes a bounded domain in $\mathbf{C}^{3 g-3}$. Let $\left\{z_{i}\right\}$ be local parameters on an open cover $S=\bigcup U_{i}$ of the Riemann surface $S$. A collection of complex valued functions $\phi_{i}$ on $U_{i}$ is a differential of type $(m, n)$ if the functions transform according to the rule

$$
\phi_{i}\left(\frac{d z_{i}}{d z_{j}}\right)^{m}\left(\overline{\frac{d z_{i}}{d z_{j}}}\right)^{n}=\phi_{j}
$$

whenever $U_{i} \cap U_{j} \neq \emptyset$. A differential of type $(2,0)$ is called a quadratic differential. A differential of type $(-1,1)$ where $\phi$ is measurable and $\|\phi\|_{\infty}<1$ is called a Beltrami differential. A homeomorphism $f$ from one Riemann surface to another is called quasiconformal if $f$ is an $L^{2}$ solution of the differential equation $\bar{\partial} f=\mu \partial f$ for some Beltrami differential $\mu$. Given a Beltrami differential $\mu$ on a Riemann surface $S$, there exists a quasiconformal map of $S$ onto another Riemann surface with complex dilation $\mu$. This map is uniquely determined up to a conformal map. Hence, a Beltrami differential on a Riemann surface $S$ determines a complex structure on $S$. To define Teichmüller space consider all quasiconformal mappings of a Riemann surface $F$ onto other Riemann surfaces. Let two maps $f_{1}$ and $f_{2}$ be equivalent when $f_{2} \circ f_{1}^{-1}$ is homotopic to a conformal map. The set of equivalence classes is Teichmüller space. In case the Riemann surface is compact each homotopy class of an orientation preserving homeomorphism contains a quasiconformal map, so this definition agrees with the previous ones. Suppose a Riemann surface $R$ is uniformized by the upper half plane. Let $G_{R}$ be the Fuchsian model of $R$. Given a Beltrami differential $\mu$ on $R$, it is possible to lift $\mu$ to $H$ in a $G_{R}$ invariant way. Extend this differential to the complex plane by setting it to zero in the complement of the upper half plane. There is a quasiconformal map $f_{\mu}$ corresponding to this extended Beltrami differential. If we require $f_{\mu}$ to fix $0,1, \infty$, then $f_{\mu}$ is unique. Note that $f_{\mu}$ is conformal in 
the lower half plane. The Schwarzian derivative of $f_{\mu}$ gives a holomorphic automorphic form of weight -4 with respect to $G_{R}$ on the lower half plane. Let $A_{2}\left(H^{*}, G_{R}\right)$ be the complex vector space of holomorphic automorphic forms of weight -4 on the lower half plane. We can define the Bers' embedding by sending $\mu$ to the Schwarzian derivative $S\left(f_{\mu_{H^{*}}}\right)$. A fundamental theorem states that the Bers' embedding realizes Teichmüller space as a bounded domain in $A_{2}\left(H^{*}, G_{R}\right)$. We thus obtain a complex structure on Teichmüller space via the Bers' embedding. The mapping class group acts as a group of biholomorphic automorphisms of Teichmüller space. Since we can realize Teichmüller space as a bounded domain in a complex vector space we can apply normality arguments to the mapping class group. For example, we can prove that the mapping class group acts properly discontinuously on Teichmüller space.

Proposition 1.11. The Teichmüller modular group acts properly discontinuously on Teichmüller space.

The proof is by contradiction. The strategy is to find a sequence $f_{n} \in \Gamma_{g}$ converging to $f$ uniformly on compact subsets of $T_{g}$. Consider the elements $h_{n}=f_{n+1}^{-1} \circ f_{n}$ converging to an element $h \in \Gamma_{g}$. Using the fact that the square of the traces of the elements of a Fuchsian group is discrete in $\mathbb{R}$, we conclude that $h_{n}$ must be in the isotropy group of some point in $T_{g}$ after some large $n$. We thus obtain an infinite sequence of elements in the isotropy group of a point in $T_{g}$. But the isotropy group of a point is isomorphic to the biholomorphic automorphisms of the Riemann surface, which is a finite group. This contradicts the fact that we had an infinite sequence. Now let us flesh out the details.

Lemma 1.12. The set of hyperbolic lengths of all closed geodesics on a closed Riemann surface $R$ of genus $g \geq 2$ is a discrete set of real numbers. Moreover, for any positive number there are at most finitely many closed geodesics with that hyperbolic length.

Proof. Suppose there exists a sequence $\left\{C_{n}\right\}_{n=1}^{\infty}$ of simple closed geodesics on $R$ with $l\left(C_{n}\right) \leq M$ for some finite positive number $M$. We derive a contradiction by showing that the Fuchsian group of covering transformations of $R$ cannot be discrete in $A u t(\mathbb{H})$. Choose a relatively compact fundamental domain for the Fuchsian group and choose elements of the group representing the closed geodesics. We thus obtain a sequence of mutually distinct elements $\gamma_{n}$ of the Fuchsian group for which $\min _{z \in \bar{F}} \rho\left(z, \gamma_{n}(z)\right) \leq M$, where $\bar{F}$ is the closure (compact by choice) of the fundamental domain. Now $\gamma_{n}$ is a normal family, hence, by choosing a subsequence if necessary, we see that they converge to an 
automorphism of $H$. Hence the Fuchsian group is not discrete. This is a contradiction.

We may conclude from this lemma that $\left\{\operatorname{tr}^{2}(\gamma): \gamma \in \Gamma\right\}$ is a discrete set of real numbers. In lemma 1.8 we proved that

$$
\operatorname{tr}(\gamma)^{2}=4 \cosh ^{2}\left(\frac{l\left(L_{\gamma}\right)}{2}\right)
$$

Since the lengths of the geodesics are discrete, so must the square of the traces of the group elements.

A final observation is that given a system of generators $\left\{g_{i}\right\}$ for a Fuchsian model of a closed Riemann surface such that $g_{1}$ has a repelling fixed point at 0 and an attractive fixed point at $\infty$ and $g_{2}$ has an attractive fixed point at 1 and a repelling fixed point at $r<0$, the generators are determined by the absolute values of the traces in

$$
\left\{g_{i}, g_{1} \circ g_{i}, g_{1}^{-1} \circ g_{i}, g_{2} \circ g_{i}, g_{2}^{-1} \circ g_{i}, g_{1} \circ g_{2} \circ g_{i},\left(g_{1} \circ g_{2}\right)^{-1} \circ g_{i}\right\}
$$

Let us return to the proof that the mapping class group acts properly discontinuously on Teichmüller space. Suppose not. Then we can find a sequence of distinct elements in the mapping class group and a sequence of points $p_{n}$ in Teichmüller space converging to a point $p$ such that

$$
f_{n}\left(p_{n}\right) \rightarrow p \in T_{g} .
$$

By the normality of the family, selecting a subsequence if necessary, we can assume that $f_{n}$ converges to $f$ uniformly on compact subsets of the Teichmüller space. Set $h_{n}=f_{n+1}^{-1} \circ f_{n}$. Observe that $h_{n}$ converges to some $h$ uniformly on compact subsets of Teichmüller space and $h$ fixes the point $p$. By translating $p$ to the identity we can assume that $h$ fixes the identity. We want to deduce that after some $n$ each $h_{n}$ fixes the identity. If we let $w_{n}$ represent $h_{n}$, then for any $g$ in the Fuchsian group we have

$$
\lim _{n \rightarrow \infty} t r^{2}\left(w_{n}^{-1} g w_{n}\right)=t r^{2}(g) .
$$

Since the traces are discrete, after some $n$ equality must hold without the limit. Since the group is determined by the traces of finitely many elements, after some $n$ each $h_{n}$ must be in the normalizer of the group. Hence $h_{n}$ is in the isotropy group of the identity for all sufficiently large $n$. This is a contradiction. We conclude that the mapping class group acts properly discontinuously on Teichmüller space.

The action of the mapping class group, however, is not fixed point free. Riemann surfaces with non-trivial biholomorphic automorphisms are fixed by suitable elements of the group. In general, the moduli space is not a manifold. However, as we observed in the beginning of 
this section, Proposition 1.11 implies that $M_{g}$ has an orbifold structure.

Remark 1.13. In this section to make the exposition more manageable I described the Teichmüller space of compact genus $g$ surfaces. In the sequel we will need to consider the case when the surface has marked points and boundary components. Let $F_{g, n}$ be a surface of genus $g$ with $n$ marked points $p_{1}, \cdots, p_{n}$. Consider triples $\left(R, q_{i},[f]\right)$ consisting of a Riemann surface $R$ with n parked points $q_{1}, \cdots, q_{n}$ and the homotopy class (relative to the $q_{i}$ ) of an orientation preserving homeomorphisms $f: R \rightarrow F$ such that $f\left(q_{i}\right)=p_{i}$. Call two triples $\left(R_{1}, q_{i}^{1},\left[f_{1}\right]\right) \cong\left(R_{2}, q_{i}^{2},\left[f_{2}\right]\right)$ equivalent iff there exists a conformal homeomorphism $h: R_{1} \rightarrow R_{2}$ such that $h\left(q_{i}^{1}\right)=q_{i}^{2}$ and $\left[f_{2} \circ h\right]=\left[f_{1}\right]$. The Teichmüller space $T_{g, n}$ is the set of equivalence classes of triples. More generally, we can allow $F$ to have boundary components. In this case we require the homotopies to fix the boundary. We obtain the Teichmüller space $T_{g, n}^{r}$. There is a corresponding mapping class group $\Gamma_{g, n}^{r}$ consisting of orientation preserving homeomorphisms of $F_{g, n}^{r}$ that fix the marked points and the boundary components. We can interpret $T_{g, n}$ from the point of view of hyperbolic geometry by considering complete hyperbolic surfaces of finite area. Here the marked points can be thought of as cusps. There is a pants decomposition provided that we allow ideal pants with one or two boundary components of length zero. The analysis in the FenchelNielsen coordinates goes through to show that the Teichmüller space is homeomorphic to $\left(\mathbb{R}^{+} \times \mathbb{R}\right)^{3(g-1)+n}$ provided that $2 g-2+n>0$.

\section{The Harer-Zagier Formula for the orbifold Euler CHARACTERISTIC OF THE MAPPING CLASS GROUP}

The main references for this section are [HZ] and [Har4].

Here we will discuss remarkable formula due to Harer and Zagier, which relates the orbifold Euler characteristic of the mapping class group $\Gamma_{1, g}$ to the values of the zeta function at the negative odd integers.

$$
\chi_{\text {orb }}(\Gamma g, 1)=\zeta(1-2 g)=-\frac{B_{2 g}}{2 g}
$$

Pick a torsion free subgroup $\Gamma^{\prime}$ of finite index in $\Gamma_{g, 1}$. Note that $\Gamma^{\prime}$ acts on Teichmüller space freely and properly discontinuously, so the quotient of Teichmüller space by $\Gamma^{\prime}$ is a manifold. Define the orbifold Euler characteristic of $\Gamma_{1, g}$ as the Euler characteristic of this manifold divided by the index of $\Gamma^{\prime}$ in $\Gamma_{1, g}$. Note that the orbifold Euler 
characteristic does not depend on the choice of $\Gamma^{\prime}$. Given a different subgroup $\Gamma^{\prime \prime}$ we can find a common subgroup $H$ of $\Gamma^{\prime}$ and $\Gamma^{\prime \prime}$ so that $H$ has finite index in $\Gamma_{1, g}$. By the multiplicativity of indexes and of the Euler characteristics of covering spaces we conclude that the orbifold Euler characteristic of $\Gamma_{1, g}$ is independent of the choice of finite index subgroup.

Suppose a group acts on a contractible cell complex respecting the cell structure of the complex. Under some finiteness conditions a theorem of Quillen gives a method for calculating the orbifold Euler characteristic of a group. Splitting the cell complex to orbits leads to a spectral sequence. Denoting the stabilizers of a representative of each orbit by $G_{p}^{i}$, we can write Quillen's formula as

$$
\chi_{\text {orb }}\left(\Gamma_{1, g}\right)=\sum_{p}(-1)^{p} \sum_{i} \chi\left(G_{p}^{i}\right) .
$$

We will now describe a contractible cell complex on which $\Gamma_{1, g}$ acts preserving the cell structure. Let $F$ be a Riemann surface of genus $g$ with a base point $q$. An arc system of rank $k$ is a collection of $k+1$ isotopy classes of curves $\left\langle\alpha_{0}, \cdots \alpha_{k}\right\rangle$ that intersect only at the base point $q$ and satisfy the non-triviality condition that none of the curves are null-homotopic and no two of the curves are homotopic to each other. Here and in what follows homotopy and isotopy should be interpreted to be relative to the base point $q$. An arc system fills the surface $F$ if all the components of the complement of the curves $F-\left\{\alpha_{i}\right\}$ are simply connected. Build a cell complex $Y$ which has a $\mathrm{k}$-cell for each rank $6 g-4-k$ arc system that fills the surface. An l-cell $\left\langle\beta_{0}, \cdots, \beta_{l}>\right.$ is a face of a k-cell $\left\langle\alpha_{0}, \cdots, \alpha_{k}>\right.$ exactly when $\left\{\beta_{i}\right\} \subset\left\{\alpha_{j}\right\}$. Observe that by the non-triviality conditions the largest possible rank for an arc system is $6 g-4$ since $6 g-3$ curves separate the surface into disjoint triangles. Also observe that an arc system of rank less than or equal to $2 g-2$ cannot fill the surface. The cell complex $Y$ that we obtain is contractible. (We will assume this result without proof.) The group $\Gamma_{1, g}$ acts on $Y$. We will compute the orbifold Euler characteristic using this action. Take a rank $n=6 g-3-p$ arc system $\left\langle\alpha_{0}, \cdots, \alpha_{n}\right\rangle$ which fills $F$. We will construct a dual graph $\Omega$ to this arc system. $\Omega$ has a vertex for each connected component of $F-\left\{\alpha_{i}\right\}$ and one edge that meets $\alpha_{i}$ transversely for each $\alpha_{i}$. Cutting the surface along this graph gives a 2 n-gon $P_{n}$ with a pairing $\tau$ of the edges so that $F \cong P_{n} / \tau$. We take the marked point to be the center of the 2 n-gon. Observe that every vertex of the dual graph has valence at least 3 . If a vertex had valence 1 , then the boundary of the connected component corresponding to that vertex would have to consist of one 
curve. However, that curve would be contractible (recall the pieces are simply connected) contradicting the assumption that none of the curves in the arc system were null homotopic. If a vertex had valence 2 , then the boundary of the connected component corresponding to that vertex would have only two curves. Those curves would have to be homotopic. This violates the other non-triviality condition. These conditions imply two conditions $\mathrm{A}$ and $\mathrm{B}$ on the way the sides of $P_{n}$ can be identified. First, no two adjacent sides of $P_{n}$ are identified (A). Otherwise, the vertex common to both would have valence 1. Second, no two adjacent sides are identified to two other adjacent sides in the reverse order (B). Otherwise, the vertex common to the pair of adjacent sides would be a vertex of valence 2 . These are the only requirements on the identification. Denote by $\lambda_{g}(n)$ the number of possible pairings of the sides of $P_{n}$ satisfying both these conditions and giving an orientable genus $g$ surface. Denote by $\mu_{g}(n)$ the number of possible pairings that satisfy only the first condition but not necessarily the second. Finally denote by $\epsilon_{g}(n)$ the number of possible pairings of the sides of $P_{n}$ that gives an orientable genus $g$ surface (no conditions imposed). Note that these three numbers satisfy the following two equations:

$$
\begin{gathered}
\epsilon_{g}(n)=\sum_{i}\left(\begin{array}{c}
2 n \\
i
\end{array}\right) \mu_{g}(n-i) \\
\mu_{g}(n)=\sum_{i}\left(\begin{array}{c}
n \\
i
\end{array}\right) \lambda_{g}(n-i)
\end{gathered}
$$

To prove equation (4) start with an arbitrary pairing on the $2 n$-gon that gives a genus $g$ surface. If two adjacent sides are identified, we can eliminate them by making their common vertex an interior point of a $2 n-2$-gon. The $2 n-2$-gon has an identification of its sides that gives a genus $g$ surface. There might still be adjacent sides which are identified. (In fact, two sides that are identified can become adjacent as a result of this process.) If we continue to eliminate the adjacent sides that are identified, we eventually obtain a $2 n-2 i$-gon with no adjacent sides identified. Observe that this process does not change the genus of the surface. All the $\mu_{g}(n-i)$ possibilities can occur for each $i$. Moreover, a particular identification of $P_{n-i} \operatorname{occurs}\left(\begin{array}{c}2 n \\ i\end{array}\right)$ times since we are free to choose any of the $\mathrm{i}$ vertices of $P_{n}$ as the vertices that become the interior points of $P_{n-i}$.

The proof of equation (5) is similar in flavor. Suppose we start with an arbitrary identification satisfying condition (A) but not necessarily condition (B). We can make a pair of edges into one edge if this pair is identified to another pair in the reverse order. We get $P_{2 n-2}$ with an 
identification of its sides, which still gives a genus $g$ surface. Continuing we eventually obtain $P_{2 n-2 i}$ with an identification that satisfies both conditions. Given an admissible identification of the sides of $P_{2 n-2 i}$ there are $\left(\begin{array}{l}n \\ i\end{array}\right)$ ways that it can occur under this reduction. Orient the boundary of $P_{2 n-2 i}$ and number its edges consecutively starting from some vertex. For each pair of identified edges take the smaller of the numbers. This leads to a sequence of numbers $1<j_{1}<\cdots<j_{n-i}$. Given any $n-i$ non negative integers that sum to $i$, we can split the side corresponding to $j_{k}$ to $m_{k}+1$ pieces. The only ambiguity is in choosing which of the $m_{1}+1$ edges becomes edge 1 in $P_{n}$. Summing the possibilities leads to equation (5).

The stabilizer of a p-cell in $Y$ corresponds to the rotational symmetries of an identification of $P_{n}$ where $n=6 g-3-p$. The stabilizer of this cell is of finite order, say $2 n / m$, with Euler characteristic $m / 2 n$. Consider pairings of the edges of $P_{n}$ that satisfy both (A) and (B). Let two pairings be equivalent if they differ by a rotation of $P_{n}$. Choose a representative of each equivalence class. If an equivalence class has order $m$, then the cells have cyclic symmetry of order $2 n / m$. Hence we can count over each possible pairing giving each pairing the weight $1 / 2 n$. Using Quillen's formula we conclude that

$$
\chi_{\text {orb }}\left(\Gamma_{1, g}\right)=\sum_{p}(-1)^{p} \sum_{i} \chi\left(G_{p}^{i}\right)=\sum_{n=2 g}^{6 g-3}(-1)^{n-1} \lambda_{g}(n) .
$$

Note that the non-triviality condition on the arc systems means that there cannot be any arc systems of rank bigger than $6 g-4$. The fact that they fill the surface implies that the rank is at least $2 g-1$. This justifies letting the sum run from $2 g$ to $6 g-3$.

Now that we have obtained an expression for the orbifold Euler characteristic of $\Gamma_{1, g}$, there remains to relate this expression to the zeta function. The main task will be to prove the equation

$$
\epsilon_{g}(n)=\frac{(2 n) !}{(n+1) !(n-2 g) !} C_{o}\left(x^{2 g},\left(\frac{x / 2}{\tanh (x / 2)}\right)^{n+1}\right)
$$

where $C o\left(x^{k}, f(x)\right)$ denotes the coefficient of $x^{k}$ in the power series expansion of $f$. It is convenient to define an auxiliary polynomial whose value at 0 is the orbifold Euler characteristic. Let

$$
\epsilon_{g}(n)=\left(\begin{array}{c}
2 n \\
n+1
\end{array}\right) F_{g}(n)
$$

If we assume the previous claim about $\epsilon_{g}(n)$, then we observe that $F_{g}(n)$ is a polynomial of degree $3 g-1$. It vanishes at -1 , hence $n+1$ 
divides it. Taking $(n-1),(n-1)(n-2), \cdots$ as our basis for polynomials we can express $F$ as

$$
F_{g}(n)=(n+1) \sum_{r=1}^{d} \frac{r !}{(2 r) !} \kappa_{g}(r)(n-1)(n-2) \cdots(n-r+1)
$$

The factor $r ! /(2 r)$ ! will simplify some of the equations that will occur later. Observe that we can express $\epsilon_{g}(n)$ as

$$
\epsilon_{g}(n)=\frac{(2 n) !}{n !} \sum_{r=1}^{d} \frac{r ! \kappa_{g}(r)}{(2 r) !(n-r) !}
$$

To complete the proof of the theorem we have to justify two assertions. First, $F_{g}(0)$ is the orbifold Euler characteristic of $\Gamma_{g, 1}$. Second, $\epsilon_{g}(n)$ is given by equation (7). Observe that the Taylor expansion of $(t / 2) / \tanh (t / 2)$ leads to the Harer-Zagier formula. We begin by verifying the first claim. Form the generating functions

$$
\begin{aligned}
& L_{g}(x)=\sum_{n \geq 0} \lambda_{g}(n) x^{n} \quad M_{g}(x)=\sum_{n \geq 0} \mu_{g}(n) x^{n} \\
& E_{g}(x)=\sum_{n \geq 0} \epsilon_{g}(n) x^{n} \quad K_{g}(x)=\sum_{n} \kappa_{g}(n) x^{n}
\end{aligned}
$$

It is possible to relate these functions to each other by using the relations between the coefficients. The following formula will be useful.

$$
\sum_{i}\left(\begin{array}{c}
2 i+k \\
i
\end{array}\right) x^{i}=\frac{1}{\sqrt{1-4 x}}\left(\frac{1-\sqrt{1-4 x}}{2 x}\right)^{k}
$$

To prove this formula observe that when $k=0$ we have the well known case $(1-4 x)^{-1 / 2}$. Let the sum on the left be $f_{k}$. Writing

$$
\left(\begin{array}{c}
2 i+k \\
i
\end{array}\right)=\left(\begin{array}{c}
2 i+k-1 \\
i
\end{array}\right)+\left(\begin{array}{c}
2(i-1)+k+1 \\
i-1
\end{array}\right)
$$

we obtain the recursion relation $f_{k-2}=f_{k-1}+x f_{k}$ valid for $k \geq 2$. For $k=1$ one has to fix the first few terms to obtain $f_{0}=2 x f_{1}+1$. The rest follows by induction. We can now obtain the relations among the 
generating functions we defined. To relate $E$ and $M$ write

$$
\begin{aligned}
E_{g}(x) & =\sum_{n} \epsilon_{g}(n) x^{n}=\sum_{n}\left(\sum_{i}\left(\begin{array}{c}
2 n \\
i
\end{array}\right) \mu_{g}(n-i)\right) x^{n} \\
& =\sum_{j \geq 0} \sum_{i}\left(\begin{array}{c}
2 i+2 j \\
i
\end{array}\right) \mu_{g}(j) x^{i+j}=\sum_{j} \mu_{g}(j) x^{j} \sum_{i}\left(\begin{array}{c}
2 i+2 j \\
i
\end{array}\right) x^{i} \\
& =\frac{1}{\sqrt{1-4 x}} M_{g}\left(\frac{1-2 x-\sqrt{1-4 x}}{2 x}\right)
\end{aligned}
$$

To relate $E$ and $K$ we write

$$
\begin{aligned}
E_{g}(x) & =\sum_{n} \frac{(2 n) !}{n !} \sum_{r=1}^{d} \frac{r ! \kappa_{g}(r) x^{n}}{(2 r) !(n-r) !}=\sum_{r=1}^{d} \frac{r ! \kappa_{g}(r)}{(2 r) !} \sum_{n} \frac{(2 n) ! x^{n}}{n !(n-r) !} \\
& =\sum_{r=1}^{d} \frac{r ! \kappa_{g}(r)}{(2 r) !} x^{r} \frac{d^{r}}{d x^{r}} \sum_{n} \frac{(2 n) ! x^{n}}{n !(n) !}=\sum_{r=1}^{d} \frac{r ! \kappa_{g}(r)}{(2 r) !} x^{r} \frac{d^{r}(1-4 x)^{-1 / 2}}{d x^{r}} \\
& =\frac{1}{\sqrt{1-4 x}} \sum_{r} \kappa_{g}(r)\left(\frac{x}{1-4 x}\right)^{r}=\frac{1}{\sqrt{1-4 x}} K_{g}\left(\frac{x}{1-4 x}\right)
\end{aligned}
$$

Finally to relate $L$ to the rest

$$
\begin{aligned}
M_{g}(x) & =\sum_{n} \sum_{i}\left(\begin{array}{c}
n \\
i
\end{array}\right) \lambda_{g}(n-i) x^{n}=\sum_{j} \lambda_{g}(j) x^{j} \sum_{i}\left(\begin{array}{c}
i+j \\
i
\end{array}\right) x^{i} \\
& =\sum_{j} \lambda_{g}(j) x^{j} \frac{1}{(1-x)^{j+1}}=\frac{1}{1-x} L_{g}\left(\frac{x}{1-x}\right)
\end{aligned}
$$

Combining these relations we obtain

$$
L_{g}(x)=\frac{1}{1+x} K_{g}(x(1+x)) .
$$

This formula allows us to relate the orbifold Euler characteristic of $\Gamma_{g, 1}$ to the value $F_{g}(0)$. We have an expression for the Euler characteristic in terms of $\lambda_{g}$. Using the previous equation we can turn this expression 
into a beta integral.

$$
\begin{aligned}
\chi_{\text {orb }}(g) & =\sum_{i} \frac{(-1)^{n-1} \lambda_{g}(n)}{2 n}=-\frac{1}{2} \int_{0}^{1} \frac{\sum_{n} \lambda_{g}(n)(-x)^{n} d x}{x} \\
& =-\frac{1}{2} \int_{0}^{1} \frac{L_{g}(-x) d x}{x}=-\frac{1}{2} \int_{0}^{1} \frac{K_{g}(-x(1-x)) d x}{x(1-x)} \\
& =\frac{1}{2} \sum_{r}(-1)^{r-1} \kappa_{g}(r) \int_{0}^{1} x^{r-1}(1-x)^{r-1} d x \\
& =\sum_{r}(-1)^{r-1} \frac{r !(r-1) ! \kappa_{g}(r)}{(2 r) !}=F_{g}(0)
\end{aligned}
$$

There remains to prove equation (7). We will first relate $\epsilon_{g}(n)$ to the number of pairs consisting of a coloring of the vertices of $P_{n}$ and an identification of the edges compatible with the coloring that gives an orientable surface. Counting the number of pairs a different way will yield an integral formula for $\epsilon_{g}(n)$. More precisely let $C(n, k)$ denote the number of pairs $(\phi, \tau)$ where $\phi$ is a k-coloring of the vertices of $P_{n}$ and $\tau$ is a compatible identification of the edges. Suppose we are given an identification that yields a genus $g$ surface. In $P_{n}$ there are $n+1-2 g$ inequivalent vertices. These can be colored arbitrarily. So given an identification that yields a genus $g$ surface there are $k^{n+1-2 g}$ possible ways to color the vertices. On the other hand, the sides can be paired to give any surface of genus between 0 and $n / 2$. We conclude

$$
C(n, k)=\sum_{0}^{n / 2} \epsilon_{g}(n) k^{n+1-2 g} .
$$

It is convenient to introduce another auxiliary function $D(n, k)$, which will be related to $C(n, k)$ by the equation

$$
C(n, k)=(2 n-1) ! ! D(n, k) .
$$

However, to make $D(n, k)$ 's connection to the zeta function more apparent it is more convenient to define it by the equality

$$
1+2 \sum_{n=0}^{\infty} D(n, k) x^{n+1}=\left(\frac{1+x}{1-x}\right)^{k} .
$$

Showing the relation between $C(n, k)$ and $D(n, k)$ will be the hard work. Once we assume this relation the main theorem follows. Differentiate both sides of the defining equation of $D(n, k)$. To extract $D(n, k)$ we can divide both sides of the resulting equation by $x^{n+1}$. 
The residue at 0 will be $(n+1) D(n, k)$. Substituting $x=\tanh (t / 2)$ we obtain

$$
(n+1) D(n, k)=\operatorname{res}_{t=0} \frac{k}{2}\left(\frac{1}{\tanh (t / 2)}\right)^{n+1} e^{k t} d t
$$

Consider the function $e^{k t}((t / 2) / \tanh (t / 2))^{n+1}$. The above residue will be $2^{n} k$ times the coefficient of $t^{n}$ of this function. $((t / 2) / \tanh (t / 2))^{n+1}$ is an even function and we know the power series expansion of $e^{k t}$. Multiplying these out and using the equation that relates $C(n, k)$ and $D(n, k)$ we obtain

$$
C(n, k)=\sum_{g=0}^{n / 2} \frac{(2 n) ! k^{n+1-2 g}}{(n+1) !(n-2 g) !} C o\left(t^{2 g},\left(\frac{t / 2}{\tanh (t / 2)}\right)^{n+1}\right) .
$$

Comparing the coefficients of $k$ in this expression and in the expression defining $C(n, k)$, we obtain equation (7). This completes the proof of the theorem that

$$
\chi_{\text {orb }}\left(\Gamma_{1, g}\right)=\zeta(1-2 g)
$$

modulo the equality $C(n, k)=(2 n-1) ! ! D(n, k)$. We now sketch a proof of this equality.

Observe that $D(n, k)$ is a polynomial of degree $k-1$ in $n$. To be precise expanding $((1+x) /(1-x))^{k}$ using the binomial coefficients theorem we see that

$$
D(n, k)=\sum_{l=1}^{k} 2^{l-1}\left(\begin{array}{l}
k \\
l
\end{array}\right)\left(\begin{array}{c}
n \\
l-1
\end{array}\right) .
$$

Suppose $C(n, k)$ could be expressed as $(2 n-1) ! ! Q(n, k)$, where $Q(n, k)$ is a polynomial of degree $k-1$ in $n$. Then we can identify $Q(n, k)$ and $C(n, k)$. Note that this statement should be the content of lemma 8.7 in Harer's C.I.M.E. notes. Recall that $C(n, k)$ was the number of pairs consisting of a coloring of the vertices of $P_{n}$ and a compatible identification of the edges. The number of compatible pairs where the coloring uses all $\mathrm{k}$ available colors at least once generates a new function $S(n, k)$. Since any pair counted in $C(n, k)$ uses $l$ different colors and since there are $\left(\begin{array}{l}k \\ l\end{array}\right)$ choices for the colors, we obtain

$$
C(n, k)=\sum_{l=0}^{k}\left(\begin{array}{l}
k \\
l
\end{array}\right) S(n, l)
$$


or equivalently

$$
\begin{aligned}
S(n, k) & =\sum_{l=0}^{k}(-1)^{k-l}\left(\begin{array}{l}
k \\
l
\end{array}\right) C(n, l)=(2 n-1) ! ! \sum_{l=0}^{k}(-1)^{k-l}\left(\begin{array}{l}
k \\
l
\end{array}\right) Q(n, k) \\
& \equiv(2 n-1) ! ! Q^{\prime}(n, k) .
\end{aligned}
$$

Observe that our assumptions about $Q(n, k)$ imply that $Q^{\prime}(n, k)$ is a polynomial of degree $k-1$ in $n$. Since there cannot be any surjective coloring of $P_{n}$ by $k$ colors if $n<k-1$ this polynomial vanishes at $0,1, \cdots k-2$, so

$$
Q^{\prime}(n, k)=\delta_{k}\left(\begin{array}{c}
n \\
k-1
\end{array}\right)
$$

where $\delta_{k}$ does not depend on $n$. We have to identify $\delta_{k}$. Since $\delta_{k}$ does not depend on $n$ but only on $k$ we can determine it in the case when $n=k-1 . S(k-1, k)$ is $k ! \epsilon_{0}(k-1)$ since the only genus that one can obtain from $P_{k-1}$ by using $k$ colors is a genus 0 surface and there are $k$ ! choices of colors for each surface. $\epsilon_{0}(k)$ is the k-th Catalan number. Putting these facts together gives $\delta_{k}=2^{k-1}$. We proved

$$
\begin{aligned}
C(n, k) & =\sum_{l=0}^{k}\left(\begin{array}{l}
k \\
l
\end{array}\right) S(n, l)=(2 n-1) ! ! \sum_{l=1}^{k-1} 2^{l-1}\left(\begin{array}{l}
k \\
l
\end{array}\right)\left(\begin{array}{c}
n \\
k-1
\end{array}\right) \\
& =(2 n-1) ! ! D(n, k)
\end{aligned}
$$

except for the assertion that $C(n, k)$ can be expressed as a polynomial of degree $k-1$ in $n$ times $(2 n-1)$ !! . To conclude the proof we exhibit $C(n, k)$ as such a polynomial.

We are going to obtain a different formula for $C(n, k)$ by first fixing a coloring, then counting all the possible ways to identify the sides compatible with that coloring. Fix an orientation of the boundary of $P_{n}$. Denote by $n_{i j}$ the number of edges that have the coloring $i-j$. First, observe that for there to be a compatible identification $n_{i j}=n_{j i}$ and $2 \mid n_{i i}$. Note that the matrix $N=\left(n_{i j}\right)$ is symmetric and the entries along the diagonal are even. Call such matrices even symmetric matrices. Once these conditions on $n_{i j}$ are satisfied for every $i, j$ the number of ways to identify the sides in a compatible way is $\prod_{i<j} n_{i j} ! \prod_{i}\left(n_{i i}-1\right) !$ !. We can express $C(n, k)$ as a sum over matrices with non-negative integer coefficients such that $\sum_{i, j} n_{i j}=2 n$. We sum the product of the number of ways $C(N)$ of coloring $P_{n}$ with $n_{i j}$ edges having color $i-j$ and the number of ways $E(N)$ of identifying the 
edges compatible with that coloring to obtain an orientable surface:

$$
C(n, k)=\sum_{N} C(N) E(N) .
$$

Using the integral representations of the delta function and the gamma function, we can express $C(n, k)$ as

$$
C(n, k)=2^{-k / 2} \pi^{-k^{2} / 2} \int_{H_{k}} \operatorname{tr}\left(Z^{2 n}\right) e^{-\frac{1}{2} \operatorname{tr}\left(Z^{2}\right)} d \nu_{H}
$$

where the integral is over $k \times k$ Hermitian matrices. Every Hermitian matrix can be diagonalized by conjugation. The diagonal matrix is unique up to a permutation. This allows to rewrite the integral as an integral over diagonal matrices. (Observe that the function we are integrating is invariant under conjugation.) Our function is easy to evaluate on diagonal matrices. One obtains the formula

$$
C(n, k)=c_{k} \int_{\mathbf{R}^{k}}\left(\sum_{i=1}^{k} t_{i}^{2 n}\right) e^{-\frac{1}{2}\left(\sum_{i=1}^{k} t_{i}^{2}\right)} \prod_{1 \leq i, j \leq k}\left(t_{i}-t_{j}\right)^{2} d t_{1} \cdots d_{t_{k}} .
$$

Using the symmetry of the integral one can integrate over $t_{1}$ to express $C(n, k)$ as a polynomial of degree $k-1$ times $(2 n-1)$ !!. This completes the proof of the theorem.

Remark: There are ways of obtaining the actual Euler characteristic of the Moduli space using the orbifold Euler characteristic. Harer and Zagier have given a generating function for the Euler characteristic of $\Gamma_{g}$. In view of the techniques Arbarello and Cornalba use, knowing the Euler characteristics of various Moduli spaces becomes important. Hence, as our knowledge about the homology groups of various moduli spaces increases, the Harer Zagier formula might have many applications. Also note that in the process of the proof we have counted the ways of identifying the sides of a polygon to obtain a genus $g$ surface. This seems to be an interesting geometric fact that is not immediately apparent.

\section{The stable Cohomology of $M_{g}$, Mumford's Conjecture}

The basic strategy in calculating the orbifold Euler characteristic of moduli space was to relate the Euler characteristic to an invariant of the mapping class group. Then using the action of the mapping class group on another suitable space one was able to calculate the Euler characteristic. In fact, Harer has used this basic strategy in many

other circumstances to obtain information about the cohomology of 
the moduli space of curves. In this section we briefly summarize some of his results without giving any proofs. For the proofs the reader may consult [Har2], Har3], Har1] and [Har4].

An important corollary of the construction of the moduli space of curves as the quotient of Teichmüller space by the action of the mapping class group is that the homology with $\mathbb{Q}$-coefficients of $M_{g}$ is isomorphic to the homology of the mapping class group. This allows Harer to compute the homology of $M_{g}$ via the homology of the mapping class group.

An initial result about the homology or more precisely the homotopy type of $M_{g, n}$ is that it has the homotopy type of a finite cell complex of dimension $4 g-4+n$ when $n>0$. This allows us to conclude that the $k$-th homology groups of $M_{g, n}$ vanish for sufficiently large values of $k$. The precise statements and proofs may be found in [Har3].

Theorem 3.1. The moduli space $M_{g, n}$ has the homotopy type of a finite cell-complex of dimension $4 g-4+n, n>0$. Using this one may deduce that

$$
\begin{aligned}
& \text { if } n>0 \text { and } k>4 g-4+n \text { and } \\
& H_{k}\left(M_{g, n}, \mathbb{Z}\right)=0 \\
& \text { if } k>4 g-5 .
\end{aligned}
$$

Problem 3.2. Determine the precise homological dimension of the moduli space of curves.

Harer's next result is that the $k$-th homology group of $M_{g, n}$ does not depend on $g$ provided $k$ is small compared to $g$. Again the details and precise statements may be found in [Har2]. One advantage of working with the mapping class group is that we can allow the Riemann surfaces to have boundaries. Let $\Sigma_{g, n}^{r}$ denote a Riemann surface of genus $g$ with $n$ marked points and $r$ boundary components. Define the mapping class group $M a p_{g, n}^{r}$ to be the homotopy classes of orientation preserving diffeomorphisms of $\Sigma_{g, n}^{r}$ that restrict to the identity on the boundary and the marked points.

There are the following inclusions between these mapping class group given by the geometric operations depicted in Figure 2.

Figure 2. The maps that occur in Harer's Stability Theorem 
(1) There is a map $\Phi: M_{a p_{g, n}^{r}}^{r} \rightarrow M_{a p_{g, n}^{r+1}}$ induced by attaching a pair of pants along one of the boundary components of $\Sigma_{g, n}^{r}$. In particular, we need to assume that $r \geq 1$ for this map to make sense.

(2) There is a map $\Psi: M a p_{g, n}^{r} \rightarrow M a p_{g+1, n}^{r-1}$ induced by attaching a pair of pants to $\Sigma_{g, n}^{r}$ along two boundary components. Here we need to assume that $r \geq 2$.

(3) Finally there is a map $\eta: M a p_{g, n}^{r} \rightarrow M a p_{g+1, n}^{r-2}$ induced by gluing two boundary components of $\Sigma_{g, n}^{r}$. We again need to assume that $r \geq 2$.

Harer's stability theorem asserts that the maps $\Phi, \Psi$ and $\eta$ induce isomorphisms on homology in a certain range.

Theorem 3.3. (1) $\Phi_{*}: H_{k}\left(\operatorname{Map}_{g, n}^{r}\right) \rightarrow H_{k}\left(M_{a p}^{r+1}\right)$ is an isomorphism if $g \geq 3 k-2$ and $r \geq 1$.

(2) $\Psi_{*}: H_{k}\left(\operatorname{Map}_{g, n}^{r}\right) \rightarrow H_{k}\left(\operatorname{Map}_{g+1, n}^{r-1}\right)$ is an isomorphism if $g \geq$ $3 k-1$ and $r \geq 2$.

(3) $\eta_{*}: H_{k}\left(\operatorname{Map}_{g, n}^{r}\right) \rightarrow H_{k}\left(\operatorname{Map}_{g+1, n}^{r-2}\right)$ is an isomorphism if $g \geq 3 k$ and $r \geq 2$.

In particular, combining these isomorphisms we see that $H_{k}\left(M_{g, n}, \mathbb{Q}\right)$ does not depend on $g$ provided $g \geq 3 k+1$. Using the universal coefficients theorem similarly we can say that $H^{k}\left(M_{g, n}, \mathbb{Q}\right)$ does not depend on $g$ provided $g \geq 3 k+1$. Moreover, the isomorphisms are compatible with cup products. This allows one to define a stable cohomology ring $H_{\text {stab }}^{*}(M, \mathbb{Q})$ of moduli spaces of curves by setting the $k$-th cohomology group to be $H^{k}\left(M_{g}, \mathbb{Q}\right)$ for $g>3 k+1$.

The first question that presents itself is to describe $H_{\text {stab }}^{*}(M, \mathbb{Q})$. Consider the tautological map

$$
\pi: M_{g, 1} \rightarrow M_{g}
$$

given by forgetting the marked point. Let $\zeta=c_{1}\left(\omega_{M_{g, 1} / M_{g}}\right)$. We obtain a collection of natural even cohomology classes on $M_{g}$ by considering $\kappa_{i}=\pi_{*}\left(\zeta^{i+1}\right)$. The celebrated Mumford conjecture states that these classes generate the stable cohomology of curves.

Theorem 3.4 (Mumford's Conjecture). The stable cohomology ring of curves is isomorphic to the polynomial algebra generated by the classes $\kappa_{i}$

$$
H_{s t a b}^{*}(M, \mathbb{Q}) \cong \mathbb{Q}\left[\kappa_{1}, \kappa_{2}, \ldots\right]
$$


One of the major achievements of the last few years has been the proof of Mumford's conjecture by the efforts of Madsen, Weiss, Ullrike, Tillman, Galatius among many others. For proofs, references and discussion consult Madsen and Weiss' paper math.AT/0212321, [MW], [MT] and Gal]. The proof is well-beyond the techniques developed in this class.

Problem 3.5. Harer's vanishing results and Mumford's conjecture allows us to understand the cohomology of $M_{g}$ in a certain range. Note however that the computation of the Euler characteristic suggests that the dimension of the cohomology groups grow more than exponentially. The fact that the Euler characteristic is often negative means that $M_{g}$ has a lot of odd cohomology. Construct odd cohomology classes on $M_{g}$. Construct cohomology classes on $M_{g}$ in general. In particular, are there constructions that would explain the more than exponential growth of the Euler characteristic? Despite the incredible efforts of many mathematicians our knowledge of the cohomology of $M_{g}$ remains fairly limited.

\section{Some small homology groups of the moduli SPACE of CURVES}

In this section we will give a rough sketch of Harer's celebrated theorem

Theorem 4.1. $H_{2}\left(\operatorname{Map}_{g, n}^{r} ; \mathbb{Z}\right)=\mathbb{Z}^{n+1} ; g \geq 5$.

We will later give an algebraic proof of this result due to Arbarello and Cornalba. By a theorem of Mumford $\operatorname{Pic}\left(M_{g, n}\right) \cong H^{2}\left(\operatorname{Map}_{g, n}\right)$, where $\operatorname{Pic}\left(M_{g, n}\right)$ denotes the Picard group of $M_{g, n}$. Hence, Harer's theorem determines the rank of the Picard group of the moduli space. In particular, when $n=0$ and $g \geq 5$ the rank of the Picard group of $M_{g}$ is one.

4.1. Preliminaries about the mapping class group. In this section, following Birman [Bir], we will outline a proof of the fact that the mapping class group of a genus g surface is generated by Dehn twists. In fact, Dehn twists on finitely many simple closed curves suffice to generate the group. Recall that a Dehn twist is the homeomorphism of the surface obtained by cutting the surface along a simple closed curve and re-gluing after a twist of $2 \pi$.

The proof is by induction on the genus of the surface. We have already encountered the base case in our discussion of the Teichmüller space of the sphere: Every orientation preserving homeomorphism of 
the sphere is isotopic to the identity. To carry out the induction step we establish that given any orientation preserving homeomorphism $h$ of the surface, there exists a sequence of Dehn twists and a meridian $m$ such that if $h$ is followed by a suitable sequence of Dehn twists, then $m$ stays fixed. By cutting the surface along $m$ we obtain a surface of genus $g-1$ with two disks $D_{1}, D_{2}$ removed. $h$ composed with the sequence of Dehn twists gives rise to a homeomorphism of the genus $g-1$ surface with the disks removed. This homeomorphism extends to a homeomorphism $\tilde{h}$ of the genus $g-1$ surface that fixes an interior point in each disc. We patch discs to the holes. $h$ is the identity on the boundary of the discs, so we can extend it to the disc. There is a natural surjective homomorphism from the mapping class group of a surface of genus $g-1$ with two marked points to the mapping class group of a surface of genus $g-1$. The kernel is a braid group that can be shown to be generated by Dehn twists. The result follows by induction. There remains to exhibit a meridian that is fixed when $h$ is followed by a suitable sequence of Dehn twists.

First, observe that if two simple closed curves are isotopic, then the Dehn twists generated by the two curves are isotopic. Next observe that if two simple closed curves $C_{1}$ and $C_{2}$ intersect at exactly one point, then there exists (up to a homeomorphism isotopic to the identity) a Dehn twist that takes one to the other. Act on $C_{1}$ by the Dehn twist generated by $C_{2}$. This adds a copy of $C_{2}$ to $C_{1}$, now follow this with a Dehn twist on $C_{1}$ with the appropriate orientation. To state the main lemma we need a definition. Two paths $p$ and $q$ have algebraically zero intersection if they intersect at exactly two points and if it is possible to orient $p$ so that $p$ has different directions with respect to a given orientation of $q$ at the points of intersections. The key lemma is:

Lemma 4.2. Let $p$ be a simple closed path and let $m$ be a simple path on a surface of genus $g$. Let $N$ be a regular neighborhood of $m$. Then there exists a path $u$ on the surface that lies in $p \cup N$, is related to $p$ by a sequence of Dehn twists and has either zero or algebraically zero intersection with $m$.

Proof. The proof is by induction on the cardinality of intersection between $p$ and $m$. If $p$ and $m$ do not intersect, then we can take $u$ to be $p$. If $p$ and $m$ intersect at exactly one point and $m$ is closed, then $p$ and $m$ are related by Dehn twists. Hence we can take $u$ to be $m$, but we isotope it slightly in the neighborhood so that it actually becomes disjoint from $m$. If $m$ is not closed, we can isotope it off $p$ by an isotopy that is the identity outside $N \cup p$. To complete the induction we assume 
that the lemma holds whenever the cardinality of intersection between $p$ and $m$ is less than $k$. We have to consider two cases. The first is, if we orient $p$ and $m$, then there are two adjacent points of intersection on $m$ with the same orientation. In this case we take two points slightly off $m$ in the neighborhood $N$ (see Figure 3) and consider a curve that goes close to $p$ and intersects $m$ once in a neighborhood. Doing a Dehn twist in this curve allows one to reduce the number of intersections. The induction hypothesis applies.

The second case we have to consider is the case when there are no two adjacent points with the same orientation. In this case choose three adjacent points of intersection on $m$ such that the middle one has different orientation then the outer ones. Choose a curve that intersects $m$ at one point in the neighborhood and is very close to $p$ elsewhere. A Dehn twist in this curve allows us to reduce the number of intersection. We are done by the induction hypothesis.

The lemma has an immediate strengthening from the case of a single $m$ to finitely many disjoint $m_{i}$. We are interested in this lemma since meridians satisfy the hypotheses.

Lemma 4.3. Let $p$ be a simple closed path and let $m_{1}, \cdots, m_{r}$ be disjoint, simple paths, then there exists a path $u$ which is related to $p$ by a sequence of Dehn twists and has zero or algebraically zero intersection with each of the $m_{i}$.

Choose mutually distinct neighborhoods of the paths $m_{i}$ and apply the previous lemma multiple times. Note that if at some step we have $p_{i}$ which has algebraically zero intersection with $m_{j}$ for $j \leq i$ repeating the process of the previous lemma may result in changing an algebraically zero intersection to one intersection. We can always eliminate that case using the technique described in the beginning of the proof of the previous lemma.

Lemma 4.4. Let $h$ be an orientation preserving homeomorphism of the genus $g$ surface, let $p$ be the image of the meridian $m_{1}$. Then there exists a simple closed curve $v$ related to $p$ by a sequence of Dehn twists such that $v$ does not intersect any of the other meridians, $v \cap m_{i}=\emptyset$ and the intersection of $v$ with curves $d_{i}$ that cut the genus $g$ surface into tori is either zero or algebraically zero. (see figure 2) Moreover, $v$ is related to $m_{1}$ by a sequence of Dehn twists. Consequently, $p$ is related to $m_{1}$ by a sequence of Dehn twists.

Proof. By the previous lemma we can choose $u$ so that the intersection of $u$ with $m_{i}$ and $d_{i}$ is either zero or algebraically zero. If $u$ intersects 
$m_{j}$, then it must also intersect $d_{j} . d_{j}$ bounds a torus. If $u$ did not intersect $d_{j}$, in this torus $u$ would either have to intersect itself or it would bound a disc. Neither can happen since the original meridian did not have these properties and these are properties invariant under a homeomorphism. Now it is not hard to push $u$ off $m_{j}$ by finding a disc that $u$ and $m_{j}$ bound. Repeating for every $j$ we obtain the desired curve $v$. To see that $v$ is related to $m_{1}$ by Dehn twists, remove the $g$ meridians to obtain a sphere with $2 g$ holes. $v$ must separate the sphere, but since $v$ is non-separating in the surface it must bound a boundary component. $v$ intersects a simple closed curve $a_{k}$ going once around a hole in the original surface. We can assume that the cardinality of intersection is one (or we can reduce it to that case by a sequence of Dehn twists and isotopies.) We conclude that $v$ is related to $a_{k}$ by a Dehn twist. Finally choosing a curve that intersects $a_{k}$ and $m_{1}$ once we see that $v$ is related to $m_{1}$ by Dehn twists. This completes the proof that the mapping class group is generated by Dehn twists.

4.2. Computation of $H_{1}(M a p)$. In this subsection using the fact that the mapping class group is generated by Dehn twists we will prove that the first homology group of the mapping class group with $\mathbb{Z}$ coefficients vanishes when the genus is bigger than 2 . First, recall the basic definitions of group homology. Let $\mathbb{Z} G$ denote the group ring and let $B$ be a right $\mathbb{Z} G$ module. The n-th homology group with coefficients in $A$ is defined to be

$$
H_{n}(G, A)=\operatorname{Tor}_{n}^{\mathbb{Z} G}(A, \mathbb{Z})
$$

where $\mathbb{Z}$ is regarded as a trivial $\mathbb{Z} G$ module. More explicitly, take a $\mathbb{Z} G$-projective resolution of the trivial $\mathbb{Z} G$ module $\mathbb{Z}$.

$$
\cdots P_{2} \rightarrow P_{1} \rightarrow P_{0} \rightarrow 0, \quad H_{0}(P) \cong \mathbb{Z}
$$

Tensor this complex over $\mathbb{Z} G$ by $A$ to obtain the complex

$$
\cdots \rightarrow P_{1} \otimes_{\mathbb{Z} G} A \rightarrow P_{0} \otimes_{\mathbb{Z} G} A \rightarrow 0
$$

The $n$-th homology group of $G$ with $A$ coefficients is the $n$-th homology group of this complex. It is a fact that this group is independent (up to canonical isomorphism) of the chosen projective resolution. It is useful to have explicit descriptions of the groups $H_{0}$ and $H_{1}$. First, observe that by the right exactness of the tensor product, we conclude that

$$
H_{0}(G, A) \cong A \otimes_{\mathbb{Z} G} \mathbb{Z} \text {. }
$$

The kernel of the map $\mathbb{Z} G \rightarrow \mathbb{Z}$ sending an element of $G$ to 1 is called the augmentation ideal $I G$ and as a free group it is generated on the set

$$
S=\{x-e \mid x \neq e \in G\}
$$


Since the $G$ action on $\mathbb{Z}$ is trivial, we can write

$$
A \otimes_{\mathbb{Z} G} \mathbb{Z}=A /(A \circ I G) .
$$

To compute $H_{1}(G, \mathbb{Z})$, we take the free resolution of $\mathbb{Z}$ given by

$$
0 \rightarrow I G \rightarrow \mathbb{Z} G \rightarrow \mathbb{Z} \rightarrow 0
$$

The long exact sequence of homology

$$
0 \rightarrow H_{1}(G, \mathbb{Z}) \rightarrow A \otimes_{\mathbb{Z} G} I G \stackrel{i}{\rightarrow} A \rightarrow H_{0}(G, \mathbb{Z}) \rightarrow 0
$$

implies that $H_{1}(G, \mathbb{Z}) \cong A \otimes_{\mathbb{Z} G} I G$. Here we used the facts that the higher homology groups of free modules vanish and that $i$ is trivial. Recalling that $G$ acts trivially on $\mathbb{Z}$ we obtain

$$
H_{1}(G, \mathbb{Z})=\mathbb{Z} \otimes_{\mathbb{Z} G} I G=I G /(I G)^{2} .
$$

The latter group is isomorphic to the quotient of $G$ by its commutator subgroup. We conclude from this discussion that the first homology group of $G$ with integer coefficients is isomorphic to the abelianization of the group. In this section whenever I omit the coefficients I mean homology with integer coefficients. Having mentioned the facts we will use from group homology, we can compute the first homology of the mapping class group.

Proposition 4.5. $H_{1}\left(M_{a p_{g, n}^{r}}^{r}\right)=0$ for $g \geq 3$ and $r, n$ arbitrary.

Proof. We will show that the abelianization of $M a p_{g, n}^{r}$ is trivial for $g \geq 3$ by exhibiting relations among the Dehn twists that generate it. We will show these relations by explicit calculation on a sphere $S_{4}$ with four discs removed. We will conclude that they also hold on $\Sigma_{g, n}^{r}$ by embedding $S_{4}$ into $\Sigma_{g, n}^{r}$, which we can do when $g \geq 3$.

Take a sphere and remove four discs. Label the boundary components as $C_{0}, \cdots, C_{3}$. Let $\tau_{i}$ denote the Dehn twist generated by a curve parallel to the boundary $C_{i}$. Let $C_{i j}$ denote a curve circling $C_{i}$ and $C_{j}$, and let $\tau_{i j}$ be the Dehn twist on $C_{i j}$. In the mapping class group the following relation holds between these Dehn twists:

$$
\tau_{0} \tau_{1} \tau_{2} \tau_{3}=\tau_{12} \tau_{13} \tau_{23}
$$

To prove this relation observe that we can cut $S_{4}$ along three arcs to obtain a disc. If we can show that the action of the Dehn twists on the right and left hand sides of equation (2) agree on these arcs, we can conclude that equality holds. This follows from the fact that any orientation preserving homeomorphism of the closed disc fixing the boundary is isotopic to the identity. For the calculation that the action on the arcs agree see Figure 4. 
This relation allows us to conclude that the mapping class group is generated by non-separating curves for $g>2$. Embed $S_{4}$ into the surface of interest such that boundary of one of the discs is the separating curve and the others are not. Then using the relation we can eliminate the disc that is separating. Any non-separating curve can be mapped onto another non-separating curve by an orientation preserving homeomorphism of the surface. Find two canonical systems of generators one system containing one of the curves, the other system containing the other curve. If we cut the surface along these systems, we get a polygonal region. We can find a homeomorphism of the boundary taking one curve to the other. This induces a homeomorphism of the surface. We thus obtain a relation between the Dehn twists of two non-separating curves differing by a homeomorphism $h$

$$
\tau_{A}=h \tau_{B} h^{-1} \text {. }
$$

It follows that the first homology group is cyclic since we can write all the generators as $\alpha_{i} \tau \alpha_{i}^{-1}$ for a fixed Dehn twist $\tau$ and some element $\alpha_{i} \in \Gamma_{g, n}^{r}$. When we abelianize, all the generators become equal. Hence, $H_{1}\left(\Gamma_{g, n}^{r}\right)$ is cyclic for $g \geq 3$. To show that $H_{1}$ is actually zero when $g \geq$ 3 , we use the fact that we can embed $S_{4}$ into our surface such that all the seven curves that we considered are non-separating. We then obtain the relation (2) among the seven Dehn twists $\alpha_{i} \tau \alpha_{i}^{-1}$. Abelianizing we see that $\tau^{4}=\tau^{3}$. In other words, the abelianization of the mapping class group is trivial. This proves the proposition.

4.3. Construction of the cut system complex. A cut system < $C_{i}>_{i=1}^{g}$ on the genus $g$ surface $F$ is the isotopy classes of a collection of $g$ disjoint, simple closed curves such that the complement of these curves $F-\left(C_{1} \cup \cdots \cup C_{g}\right)$ remains connected. There is no ordering or orientation on the curves. Observe that in general there will be infinitely many cut systems on a given surface. Given two isotopy classes of curves define $I\left(C, C^{\prime}\right)$ as the minimum number of intersections among representatives intersecting transversely.

We start building a cell complex $X$. X has one vertex for each cut system on $F$. We attach a one cell between vertices that represent cut systems that differ by a simple move. A cut system differs from another cut system by a simple move if the two cut systems differ in only one isotopy class and for these $I\left(C_{i}, C_{i}^{\prime}\right)=1$. For example, on the torus a loop going around the hole once represents a cut system and a loop going around the handle represents another cut system. These cut systems differ by a simple move. There are three basic cycles of simple moves. (See Figure 5) We adjoin a 2-cell each time one of these 
basic cycles of simple moves occurs. Usually when writing cycles, we omit any isotopy class that remains unchanged. Hatcher and Thurston [HT] proved that the resulting cell complex is connected and simply connected.

The main idea of the proof is to realize cut systems as maximal trees in a graph, where the vertices of the graph correspond to the critical points of a $C^{\infty}$ function and the edges correspond to the connected components of the function's level sets. Then drawing paths in the space of $C^{\infty}$ functions they are able to show that $X$ is connected. Using a careful analysis of how non-degenerate critical points can change if a family of functions has a specified type of degenerate critical point, they show that one can contract any loop. Since the details are involved we will omit them here.

The mapping class group $\Gamma$ acts on the complex $X$ by

$$
[w]<C_{i}>=<w\left(C_{i}\right)>\text {. }
$$

Observe that since the cells are determined solely by configurations of simple closed curves on $F$, this action extends to the whole complex. Unfortunately the cell complex $X$ is too large to work with. The next step is to select a Map invariant simply connected subcomplex of $X$ whose combinatorics we can control.

The definition is complicated. First, among the two cells corresponding to the $R_{1}$ cycles we pick a subset corresponding to the Map orbit of the cycles where the changing isotopy classes correspond to $\alpha_{1}, \beta_{1}, \gamma_{i}$ and the fixed ones correspond to $\alpha_{i}, 2 \leq i \leq g$. (See Figures 6 and 7.) We take all the two cells corresponding to $R_{2}$ cycles. Finally, we take the $M a p$ orbit of the $R_{3}$ cycle shown in the figure. By definition this subcomplex $Y_{2}$ is invariant under the action of the mapping class group. The main theorem is that $Y_{2}$ like $X$ is simply connected. The proof proceeds by showing that $Y_{2}$ has enough cells of each type so that when contracting any loop contained in $Y_{2}$ one can stay within $Y_{2}$ and does not have to use any other cells in $X$. Finally, by adding two types of three cells Harer constructs a 3-complex $Y_{3}$. See figures for a description of these three cells. We thus obtain a simply connected 3 -complex on which the mapping class group acts. The construction has been carried out in such a way that the action of the mapping class group decomposes into orbits since an orientation preserving homeomorphism cannot take a specific type of curve configuration to another. Moreover, we have a precise description of an element in each orbit. This allows us to compute stabilizers and compute homology groups. 
4.4. The calculation of $H_{2}(\mathrm{Map})$. Until we explicitly remove the assumptions, we will assume that $g \geq 5, n=0$ and $r \geq 1$. We will often write $M a p$ instead of $M a p_{g, n}^{r}$. Let $B$ be a CW complex and a $K($ Map, 1$)$. In other words, $B$ has trivial higher homotopy groups and $\pi_{1}(B)=$ Map. Let $E$ be the universal cover of $B$. Consider the fiber product $\Delta=E \times_{M a p} Y_{3}$. Recall that the fiber product is the quotient of the Cartesian product under the equivalence relation $(e g, y) \sim(e, g y)$. There is a natural projection from $\Delta$ to $B$ given by $\tilde{p}(e, y)=p(e)$, where $p$ is the projection map from $E$ to $B$. The fiber of this projection is $Y_{3}$. Since $Y_{3}$ is simply connected and $B$ is a $K($ Map, 1$)$ the homotopy exact sequence for a fibration allows us to conclude that $\pi_{1}(\Delta)=$ Map. Recall that we are trying to prove the statement that $H_{2}\left(M a p_{g, n}^{r}\right) \cong \mathbb{Z}^{n+1}$ for $g \geq 5$. A corollary of the Cartan-Leray spectral sequence establishes the existence of an exact sequence

$$
H_{3}(M a p) \rightarrow H_{2}(\tilde{\Delta}) \rightarrow H_{2}(\Delta) \stackrel{\phi}{\rightarrow} H_{2}(M a p) \rightarrow 0 .
$$

Here $\tilde{\Delta}$ denotes the universal cover of $\Delta$ and all the homology groups are with integer coefficients. Harer computes the image of $\phi$ in the above sequence as $\mathbb{Z}$. Since the map is surjective this shows that $\mathrm{H}_{2}(\mathrm{Map})$ is $\mathbb{Z}$.

Using the cellular chain complexes we have for $Y_{3}$ and $E$ we can obtain a cellular complex for $\Delta$. Let $\left(C_{*}, \partial_{*}^{C}\right)$ be the cellular chain complex of $Y_{3}$ and $\left(K^{*}, \partial_{*}^{K}\right)$ be the cellular chain complex of $E$. Form the tensor product of these chain complexes over the group ring of Map. Denote the resulting chain complex by $\left(M_{*}, \partial_{*}^{M}\right)$. Explicitly, $M$ is given by

$$
M_{k}=\oplus_{i+j=k} C_{i} \otimes_{\mathbb{Z M a p}} K_{j} .
$$

As usual the differential is

$$
\partial_{k}^{M}=\left(\oplus\left(\partial_{i}^{C} \otimes_{\mathbb{Z M a p}} 1_{K_{k-i}}\right)\right)+\left(\oplus\left(1_{C_{i}} \otimes_{\mathbb{Z} M a p}(-1)^{i} \partial_{k-i}^{K}\right)\right)
$$

There is a natural filtration on the chain complex $\left(M_{*}, \partial_{*}^{M}\right)$ given by

$$
F_{p}\left(M_{k}\right)=\oplus_{i+j=k, i \leq p} C_{i} \otimes_{\mathbb{Z M a p}} K_{j} .
$$

This gives rise to a spectral sequence which abuts to

$$
E_{p, q}^{\infty}=\frac{F_{p} H_{p+q}(\Delta)}{F_{p-1} H_{p+q}(\Delta)},
$$

where $F_{p}$ is the filtration of $H_{*}(\Delta)$ obtained by taking the images of $H_{p+q}\left(E \times_{\Gamma} Y_{p}\right)$ in $H_{p+q}(\Delta)$ arising from the inclusion of $E \times_{M a p} Y_{p}$ in $\Delta$. 
The main observation is that Map cannot take a given cell of $Y_{3}$ to any arbitrary cell of $Y_{3}$. Map acts transitively on the zero cells. This follows from the fact that $Y_{2}$ is connected. We can choose a path along the one skeleton going from one cut system to another. As we discussed above, there is a sequence of Dehn twists that takes a curve $\alpha$ to a curve $\beta$ when $\alpha$ intersects $\beta$ exactly once. This sequence does not affect any of the other curves in the cut system. Finally, any $R_{2}$ type cell can be taken to any other one by Map. Using this information we can obtain a description of the decomposition of $C_{p}$, the p-th graded piece in the cell complex of $Y$ into orbits of Map. For zero-cells we only need to take the zero-cell $\sigma_{0}$ corresponding to $\left\langle\alpha_{1}, \alpha_{2}, \cdots, \alpha_{g}\right\rangle$. To make the notation less cumbersome we omit the $\alpha_{i}$ that do not change from the notation. For one cells we only need to take the 1-cell $\sigma_{1}$ given by $\left\langle\alpha_{1}\right\rangle-\left\langle\beta_{1}\right\rangle$. For the two cells of type $R_{1}$ we need the $\sigma_{2}^{i}$ corresponding to $\left\langle\alpha_{1}\right\rangle-\left\langle\beta_{1}\right\rangle-\left\langle\gamma_{i}\right\rangle-\left\langle\alpha_{1}\right\rangle$ for each $\gamma_{i}, 1 \leq i \leq N$. For the two cell of type $R_{2}$ we need to include $\sigma_{2}^{N+1}$ corresponding to $\left\langle\alpha_{1}, \alpha_{2}>-<\beta_{1}, \alpha_{2}>-<\beta_{1}, \beta_{2}>-<\alpha_{1}, \beta_{2}\right\rangle$. Finally, for the 2-cell of type $R_{3}$ take $\sigma_{2}^{N+2}$. (See figure 7) For our two types of 3 -cells take $\sigma_{3}^{1}$ and $\sigma_{3}^{2}$ as pictured.

The action of Map on $C_{p}$ splits as

$$
C_{p}=C\left(\sigma_{p}^{1}\right) \oplus \cdots \oplus C\left(\sigma_{p}^{n_{p}}\right)
$$

where $C\left(n_{p}^{i}\right)$ denotes the Map orbit of the p-cell $\sigma_{p}^{i}$. Let $\Gamma_{p}^{i}$ denote the stabilizer of $\sigma_{p}^{i}$. Then we can view $C_{p}^{i}$ as

$$
C_{p}^{i}=\mathbb{Z} M a p \otimes_{\mathbb{Z} \Gamma_{p}^{i}} \mathbb{Z} .
$$

This allows us to describe the $E^{1}$ term of the spectral sequence as

$$
E_{p, q}^{1}=\oplus_{i} H_{q}\left(\Gamma_{p}^{i},<\sigma_{p}^{i}>\right) .
$$

Recall that $H_{0}$ was given as $\mathbb{Z} \otimes_{\mathbb{Z} \Gamma_{p}^{i}}<\sigma_{p}^{i}>$. To compute the various $H_{0}$ we need to know how $\Gamma_{p}^{i}$ acts on $<\sigma_{p}^{i}>$. It is clear that $\Gamma_{0}$ acts trivially on the single point $\left\langle\sigma_{0}>\right.$. We conclude that $H_{0}\left(\Gamma_{0},<\sigma_{0}>\right) \cong \mathbb{Z}$. $\Gamma_{1}$ can interchange $\alpha_{1}$ and $\beta_{1}$, so it contains an element that reverses the orientation of the 1-cell $\left\langle\sigma_{1}\right\rangle$. From this we can conclude that $H_{0}\left(\Gamma_{1},<\sigma_{1}>\right) \cong \mathbb{Z} / 2 \mathbb{Z}$ since $1 \otimes_{\mathbf{Z} \Gamma_{0}} m=1 \otimes_{\mathbb{Z} \Gamma_{0}}-m$ because of the flip. Harer observes (?) that $\Gamma_{2}^{i}$ for $1 \leq i \leq N$ acts trivially on $\left\langle\sigma_{2}^{i}\right\rangle$. It follows that $H_{0}\left(\Gamma_{2}^{i},<\sigma_{2}^{i}>\right)=\mathbb{Z} . \Gamma_{2}^{N+1}$ contains an orientation reversing element given by switching $\alpha_{1}$ and $\beta_{1}$. We conclude that $H_{0}\left(\Gamma_{2}^{N+1},<\sigma_{2}^{N+1}>\right) \cong \mathbb{Z} / 2 \mathbb{Z} . \Gamma_{2}^{N+2}$ and $\Gamma_{3}^{1}$ act trivially on $<\sigma_{2}^{N+2}>$ and $\left\langle\sigma_{3}^{1}\right\rangle$, respectively. Hence their homology groups are isomorphic 
to $\mathbb{Z} . \Gamma_{3}^{2}$ on the other hand has an orientation reversing element, hence its homology group is $\mathbb{Z} / 2 \mathbb{Z}$. This allows us to determine $E_{p, 0}^{2}$ as

$$
E_{0,0}^{2} \cong \mathbb{Z}, E_{1,0}^{2}=E_{3,0}^{2}=0, E_{2,0}^{2} \cong \mathbb{Z}^{N} \oplus \mathbb{Z} / 2 \mathbb{Z}
$$

The next step is to describe the subgroup $\hat{\Gamma}_{0}$ of $\Gamma_{0}$ that fixes the curves which determine $\sigma_{0}$ pointwise. There is an explicit description of $\hat{\Gamma}_{0}$. $\hat{\Gamma}_{0} \cong P_{2 g+r-1} \times \mathbb{Z}^{g+r-1}$. $\hat{\Gamma}_{0}$ is isomorphic to the direct product of the pure braid group on $2 g+r-1$ strings and the free abelian group generated by Dehn twists on the $\alpha_{i}$ and on $r-1$ curves parallel to the $r-1$ boundary components $\Delta_{r-1}$. On the other hand, the group of symmetries of $\sigma_{0}$ is the group of signed permutations $\pm \Sigma_{g}$ on $g$ elements since the $\alpha_{i}$ can be permuted among each other and their orientations might be reversed. In other words, there is a short exact sequence

$$
1 \rightarrow \hat{\Gamma}_{0} \rightarrow \Gamma_{0} \rightarrow \pm \Sigma_{g} \rightarrow 1
$$

Given a short exact sequence of groups there is a spectral sequence, the Lyndon-Hochschild-Serre spectral sequence, which relates the homology of the groups.

Theorem 4.6 (Lyndon-Hochschild-Serre). Given a short exact sequence of groups

$$
1 \rightarrow K \rightarrow G \rightarrow Q \rightarrow 1
$$

and $a \mathbb{Z} G$ module $M$, there exists a first quadrant spectral sequence with

$$
E_{p, q}^{2}=H_{p}\left(Q, H_{q}(K, M)\right)
$$

and converging strongly to $H_{*}(G, M)$.

A corollary of the spectral sequence is the existence of an exact sequence

$$
H_{2}(G) \rightarrow H_{2}(Q) \rightarrow K /[G, K] \rightarrow H_{1}(G) \rightarrow H_{1}(Q) \rightarrow 0
$$

Applying this exact sequence to our short exact sequence allows us to conclude that $H_{1}\left(\Gamma_{0}\right) \cong \mathbb{Z}^{N-1} \oplus \mathbb{Z} / 2 \mathbb{Z}$. Here one uses an explicit presentation of the pure braid group to compute $H_{1}\left(\hat{\Gamma}_{0}\right) . H_{1}\left(G_{0}\right)$ is the Klein 4 group. One can see this by abelianizing $\pm \Sigma_{g}$. This computation also identifies $E_{2,0}^{3}$ of our original spectral sequence as $\mathbb{Z}$. To complete the computation Harer identifies $\phi\left(F_{0}\left(H_{2}(\Delta)\right)\right)$ and $\phi\left(F_{1}\left(H_{2}(\Delta)\right)\right)$ as 0 , where $\phi$ is the map whose image we are trying to identify and $F_{p}\left(H_{2}(\Delta)\right)$ is the filtration described in the beginning of this section. This proves that $\phi: H_{2}(\Delta) \rightarrow H_{2}(M a p)$ is surjective.

There remains to remove the restrictions on the number of marked points and the number of boundary components. From now on we 
remove the global assumptions we made in the beginning of the section. There is an exact sequence that relates $M a p_{g, n}^{r}$ to $M a p_{g, n+1}^{r-1}$ when $r \geq 1$. Attach a disc to the $r$-th boundary component and make its center a marked point. Then $M a p_{g, n}^{r}$ surjects onto $M a p_{g, n+1}^{r-1}$ since any orientation preserving homeomorphism restricted to the added disc is isotopic to the identity with an isotopy that fixes the center. The kernel of the map is generated by a Dehn twist parallel to the boundary of the $r$-th component. We obtain the exact sequence

$$
1 \rightarrow \mathbb{Z} \rightarrow \operatorname{Map}_{g, n}^{r} \rightarrow \operatorname{Map}_{g, n+1}^{r-1} \rightarrow 1
$$

The Lyndon-Hochschild-Serre spectral sequence allows Harer to conclude inductively that $H_{2}\left(M a p_{g, n}^{r}\right) \cong \mathbb{Z}^{n+1}$. This settles the theorem except when the surface has no boundary components and no marked points. In this case we have to use a different exact sequence instead. There is a surjection from $M a p_{g, 1}$ to $M a p_{g}$ obtained by forgetting the marked point. (We omit the indices that are equal to 0.) This is a surjection since any orientation preserving homeomorphism is isotopic to one that fixes the marked point. The kernel of the map is isomorphic to the fundamental group of the surface. We obtain an exact sequence

$$
1 \rightarrow \pi_{1}\left(F_{g}\right) \rightarrow \operatorname{Map}_{g, 1} \rightarrow \operatorname{Map}_{g} \rightarrow 1
$$

and the Lyndon-Hochschild-Serre spectral sequence becomes available as a tool.

\section{REFERENCES}

[Ab] W. Abikoff. The real analytic theory of Teichmüller space, volume 820 of Lecture Notes in Mathematics. Springer, Berlin, 1980.

[Bir] J. S. Birman. Braids, links, and mapping class groups. Princeton University Press, Princeton, N.J., 1974. Annals of Mathematics Studies, No. 82.

[Gal] S. Galatius. Mod $p$ homology of the stable mapping class group. Topology 43(2004), 11051132.

[Har1] J. Harer. The second homology group of the mapping class group of an orientable surface. Invent. Math. 72(1983), 221-239.

[Har2] J. L. Harer. Stability of the homology of the mapping class groups of orientable surfaces. Ann. of Math. (2) 121(1985), 215-249.

[Har3] J. L. Harer. The virtual cohomological dimension of the mapping class group of an orientable surface. Invent. Math. 84(1986), 157-176.

[Har4] J. L. Harer. The cohomology of the moduli space of curves. In Theory of moduli (Montecatini Terme, 1985), volume 1337 of Lecture Notes in Math., pages 138-221. Springer, Berlin, 1988

[HZ] J. L. Harer and D. Zagier. The Euler characteristic of the moduli space of curves. Invent. math. 85(1986), 457-485.

[HT] A. Hatcher and W. Thurston. A presentation for the mapping class group of a closed orientable surface. Topology 19(1980), 221-237.

[IT] Y. Imayoshi and M. Taniguchi. An Introduction to Teichmüller Spaces. Springer-Verlag, 1992.

[Le] O. Lehto. Univalent functions and Teichmüller spaces. Springer-Verlag, 1987.

[MT] I. Madsen and U. Tillmann. The stable mapping class group and $Q\left(\mathbb{C}_{+}^{\infty}\right)$. Invent. Math. 145(2001), 509-544. 
[MW] I. Madsen and M. Weiss. The stable mapping class group and stable homotopy theory. In European Congress of Mathematics, pages 283-307. Eur. Math. Soc., Zürich, 2005. 